(C) Masson, Paris, 1980.

\title{
L'effet du repas sanguin sur la production de phéromone par les femelles de C. nubeculosus
} (Diptera, Ceratopogonidae)

\author{
par M.-T. ISMAIL et M. KREMER \\ Institut de Parasitologie et Pathologie Tropicale, 3, rue Koeberlé, F 67000 Strasbourg, \\ Directeur: $P$ 'M. Kremer
}

RESUME. Dans une étude précédente, les auteurs ont montré que les femelles vierges de $C$. nubeculosus secrètent une phéromone sexuelle qui attire les mâles de la même espèce et qui stimule l'accouplement.

Les auteurs pensent que plusieurs facteurs physiologiques pourraient modifier cette sécrétion. Ils étudient dans ce travail l'effet du repas sanguin sur la sécrétion de phéromone chez les femelles vierges.

Ils constatent que la sécrétion de phéromone n'est pas modifiée pendant le repas sanguin mais diminue dans les heures qui suivent.

Cette diminution continue jusqu'à 24 heures après le repas sanguin pendant que débute l'ovogénèse.

48 heures après le repas, lorsque les œufs sont mûrs, ils assistent à une augmentation de la sécrétion de phéromone; augmentation plus nette encore pendant la période de ponte qui a lieu 3 à 4 jours après le repas sanguin. Ensuite cette sécrétion diminue.

Effect of blood meal on the secretion of pheromone by females of C. nubeculosus (Diptera, Ceratopogonidae).

SUMMARY. In a previous study, we showed that virgin females of C. nubeculosus secrete a sex pheromone which attracts males of the same species and stimulates mating. We consider that a number of physiological factors may be capable of modifying this secretion. In this study, we have investigated the effect of a blood meal on secretion of the pheromone by virgin females. No change in secretion

Accepté le 14 mars 1980. 
of the pheromone was observed during the blood meal, but a decrease was evident during the next few hours. This decrease continues up to 24 hours after the blood meal, by which time oogenesis has started. At 48 hours after the blood meal, when the eggs are mature, secretion of the pheromone increases. An even more pronounced increase is observed during the period of oviposition, occurring 3 to 4 days after the blood meal. Secretion of the pheromone subsequently diminishes.

\section{I. - Influence du repas sur l'effet stimulant de la phéromone}

Nous avons montré précédemment que les femelles de Culicoides nubeculosus secrètent une phéromone qui stimule l'accouplement et qui attire les mâles de la même espèce (Kremer et coll.). Dans un premier temps, nous avons précisé à quel moment de leur vie, les Culicoides étaient le plus actif sexuellement, ensuite, en nous servant de cet acquis, nous avons pu contrôler que la rencontre des sexes dépendait bien de l'émission d'une phéromone et non du hasard.

Les observations de l'effet stimulant l'accouplement ont été fondées sur le comptage du nombre d'accouplements se produisant dans un bocal. Quelquefois, l'accouplement s'achève après une ou plusieurs minutes mais il est fréquent que les couples se séparent sans avoir copulé (Downes 1955). Ainsi, avons-nous défini une unité : $\mathrm{N} / \mathrm{M} / \mathrm{H}$, nombre d'accouplements par mâle et par heure (Kremer, Ismail) en comptant les vrais et les faux accouplements. Il était probable que la sécrétion de phéromone est un phénomène variable dans la vie des femelles.

Le $\mathrm{N} / \mathrm{M} / \mathrm{H}$ a été suivi en fonction de l'âge des femelles et en fonction de l'effet du repas sanguin. Dans les deux cas, nous avons travaillé avec des femelles vierges.

\section{Matériel et méthode.}

Le matériel et la méthode que nous avons utilisés sont les mêmes que ceux décrits ailleurs (Kremer et coll.) pour mettre en évidence une phéromone chez la femelle de $C$. nubeculosus: 4 chambres munies chacune d'un tube pour l'arrivée de l'air et un tube pour son évacuation.

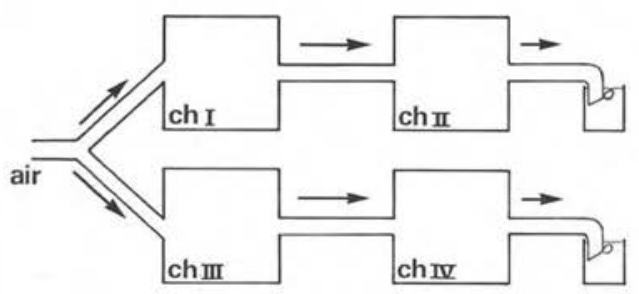

Fig. 1. Montage expérimental pour la mise en évidence de la phéromone chez la femelle de Culicoides nubeculosus. 
Les tubes ont un diamètre très petit pour ne pas laisser passer les Culicoides. Un générateur d'air du type de ceux utilisés pour l'oxygénation des aquariums, des tubes en téflon s'adaptant sur les tubes des bocaux et sur le générateur d'air, des pinces du type de celles utilisées pour régler le débit des perfusions, constituent le montage réalisé selon la figure 1.

Le flux d'air produit par le générateur est divisé en deux par un branchement en $\mathrm{Y}$, de façon aussi égale que possible. Chacune des dérivations amène 1'air successivement dans une première chambre puis dans une seconde chambre, et s'échappe finalement par un tuyau. Ce tuyau plonge dans un récipient rempli d'eau de façon à évaluer le débit par comptage des bulles d'air dans un temps donné.

Les chambres 1 et 3 servent à produire une éventuelle phéromone, l'une en activité et l'autre comme témoin.

Les chambres 2 et 4 reçoivent le flux d'air provenant des chambres précédentes et éventuellement porteur de phéromone, et servent à étudier son effet. Les expériences ont lieu à l'insectarium à $27^{\circ} \mathrm{C}, 70$ à $80 \%$ d'humidité relative et à éclairage constant. Les insectes sont obtenus par élevages traditionnels (Boorman 1974). Les observations réalisées dans les chambres 2 et 4 sont des comptages de copulation et sont exprimées par le $\mathrm{N} / \mathrm{M} / \mathrm{H}$.

\section{L'exploitation des résultats.}

Nous avons procédé, pour chaque expérience, à 5 comptages des vrais et faux accouplements.

Chaque comptage durant 5 minutes; puis nous avons calculé le $\mathrm{N} / \mathrm{M} / \mathrm{H}$ de chaque comptage et une moyenne a été établie pour ces cinq comptages.

Le résultat est exprimé par le rapport suivant :

$$
\frac{\text { La moyenne de } \mathrm{N} / \mathrm{M} / \mathrm{H} \text { de l'expérience }}{\text { La moyenne de } \mathrm{N} / \mathrm{M} / \mathrm{H} \text { de témoins }}=\frac{\mathrm{E}}{\mathrm{T}}
$$

Donc, l'accroissement du rapport exprime l'accroissement des accouplements dans l'expérience (sous l'effet de la phéromone) par rapport au témoin.

\section{A. - La sécrétion de la phéromone pendant le repas sanguin chez C. nubeculosus.}

Pour rechercher l'émission de phéromone pendant le repas sanguin, nous avons mis dans la chambre 1 , une souris de 3 jours, endormie, avec 80 femelles de $C$. nubeculosus vierges et dans la chambre 3 , une souris de même âge, endormie, comme témoin. La chambre 1 est reliée à la chambre 2, la chambre 3 à la 4 .

Dans chacune des chambres 2 et 4 , sont placés 15 femelles de 2 jours et 15 mâles de 1 jour. 
Nous avons compté les $\mathrm{N} / \mathrm{M} / \mathrm{H}$ avant le repas sanguin et pendant le repas, soit $\mathrm{E}$ pour la chambre 2 et $\mathrm{T}$ pour la chambre 4 . Les résultats sont donnés sur le tableau I.

Tableau I. Accroissement du nombre des accouplements dans ia chambre de l'expérience (2) sous l'effet du flux d'air venant de 80 femelles vierges, par rapport au témoin chambre 4. avant et pendant le repas sanguin.

$\mathrm{E}=$ la moyenne de $\mathrm{N} / \mathrm{M} / \mathrm{H}$ de l'expérience

$\mathrm{T}=$ la moyenne de $\mathrm{N} / \mathrm{M} / \mathrm{H}$ de témoin.

\begin{tabular}{ccc}
\hline Exp. $\mathrm{n}^{\circ}$ & E/T avant le repas & E/T pendant le repas \\
\hline 1 & 3,2 & 2,4 \\
\hline 2 & 2,8 & 3,1 \\
\hline 3 & 3,3 & 2,2 \\
\hline 4 & 2,2 & 2 \\
\hline moyenne & 2,9 & 2,4 \\
\hline
\end{tabular}

En conclusion, le repas sanguin ne semble pas modifier la sécrétion de phéromone.

B. - La sécrétion de phéromone dans les heures qui suivent le repas sanguin.

Dans la chambre 1 sont placées 80 à 90 femelles vierges de deux jours à jeun. La chambre 3 est vide.

Quinze couples de C. nubeculosus (mâles de 1 jour, femelles de 2 jours) sont placés dans les chambres d'observation 2 et 4 .

Le flux d'air est établi et les $\mathrm{N} / \mathrm{M} / \mathrm{H}$ sont notés plusieurs fois. Puis les femelles de la chambre 1 sont gorgées sur souris et l'expérience est reprise, les $\mathrm{N} / \mathrm{M} / \mathrm{H}$ sont notés à nouveau. Nous avons fait cette expérience 6 fois de suite avec des insectes différents.

Pour les quatre premières expériences, les 15 couples des chambres 2 et 4 n'ont pas été changé ; par contre, pour les deux dernières expériences, les 15 couples ont été changés après la $1^{\mathrm{re}}$, la $3^{\mathrm{e}}$ et la $6^{\mathrm{e}}$ heure du repas sanguin, car il arrive que plusieurs insectes meurent avant la $6^{\mathrm{e}}$ heure de l'expérience, peut-être parce qu'ils sont enfermés dans un petit espace, ou à cause de l'absence de nourriture.

Nous avons fait pour chaque expérience 5 à 10 comptages $(\mathrm{N} / \mathrm{M} / \mathrm{H})$ des accouplements. Les comptages ont été effectués par plusieurs personnes, quelques comptages ont été faits en aveugle. Les résultats sont calculés comme il est indiqué plus haut et exprimés en $\frac{E}{T}$, après avoir établi la moyenne de $N / M / H$ de l'expérience (E) et la moyenne de $\mathrm{N} / \mathrm{M} / \mathrm{H}$ de témoin $(\mathrm{T})$. 
Tableau II. Accroissement de l'accouplement dans la chambre d'expérience (2) sous l'effet $d u$ flux d'air venant de (80-90) femelles vierges par rapport au témoin, avant le repas et dans les heures qui suivent le repas sanguin.

$\mathrm{E}=$ la moyenne de $\mathrm{N} / \mathrm{M} / \mathrm{H}$ de l'expérience

$\mathrm{T}=$ la moyenne de $\mathrm{N} / \mathrm{M} / \mathrm{H}$ de témoin.

\begin{tabular}{|c|c|c|c|c|c|c|c|c|}
\hline Exp. $\mathrm{n}^{\circ}$ & $\frac{E}{T}$ & $\begin{array}{l}\text { avant le } \\
\text { repas }\end{array}$ & & $\frac{E}{T}$ & 1 heure & $\frac{E}{T}$ & 3 heures & $\frac{\mathrm{E}}{\mathrm{T}} \quad 6$ heures \\
\hline 1 & & 2,5 & \multirow{7}{*}{ 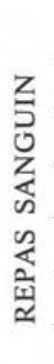 } & & 1,9 & & 1,3 & 1,2 \\
\hline 2 & & 3,9 & & & 3 & & 1,5 & 1,5 \\
\hline 3 & & 2,3 & & & 1,6 & & 1,3 & 1 \\
\hline 4 & & 2,5 & & & 1,7 & & 1,4 & 1,3 \\
\hline 5 & & 2,1 & & & 1,7 & & 1,5 & 1 \\
\hline 6 & & 4,8 & & & 2,3 & & 1,5 & 1,1 \\
\hline moyenne & & 3 & & & 2 & & 1,4 & 1,2 \\
\hline
\end{tabular}

Nous pouvons exprimer le résultat du tableau II selon la courbe représentée sur la figure 2.

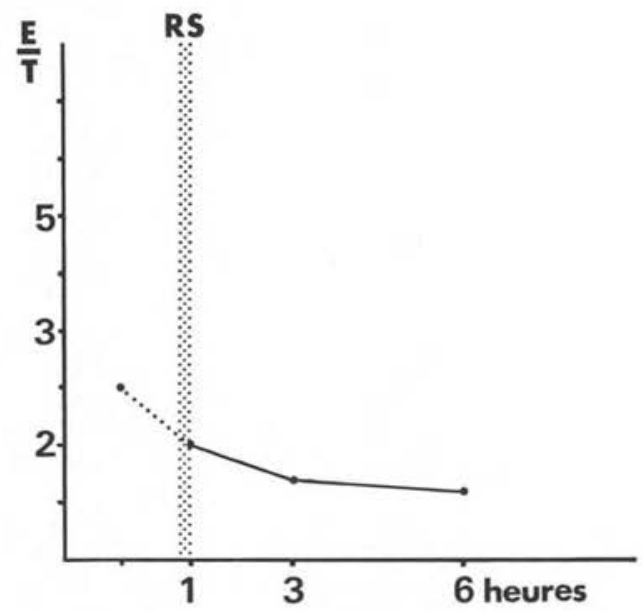

Fig. 2. Chute de la sécrétion de phéromone après le repas sanguin $(\mathrm{E}=\mathrm{la}$ moyenne de $\mathrm{N} / \mathrm{M} / \mathrm{H}$ de l'expérience, $\mathrm{T}=$ la moyenne de $\mathrm{N} / \mathrm{M} / \mathrm{H}$ de Témoins, R.S. = repas sanguin). 
Nous constatons une chute progressive de l'effet stimulant du flux d'air passant sur les femelles gorgées.

C. - La sécrétion de phéromone pendant la période de ponte.

La sécrétion de phéromone a été étudiée sur des femelles non fécondées afin de séparer l'influence de l'ovogenèse de celui de la fécondation. Nous avons suivi la sécrétion de phéromone jusqu'au $9^{e}$ jour de la vie des femelles vierges nourries en la comparant avec une série de femelles vierges à jeun et qui servent de témoin. Ces femelles à jeun ne forment pas d'œufs. 80 femelles vierges sont placées dans la chambre 1,80 femelles vierges qui prennent un repas sanguin au $2^{\mathrm{e}}$ jour de leur vie imaginale sont placées dans la chambre 3 .

15 couples de $C$. nubeculosus sont placés dans les chambres d'observation 2 et 4 . Ils ont 24 heures et sont changés à chaque observation. Sont reliées entre elles les chambres 1 et 2 ainsi que 3 et 4 .

Nous avons établi le flux d'air. Le $\mathrm{N} / \mathrm{M} / \mathrm{H}$ a été noté plusieurs fois par jour.

Nous avons refait cette expérience 4 fois avec 4 séries d'insectes. Les résultats sont donnés par les courbes de la figure 3 reproduisant les résultats du tableau III.

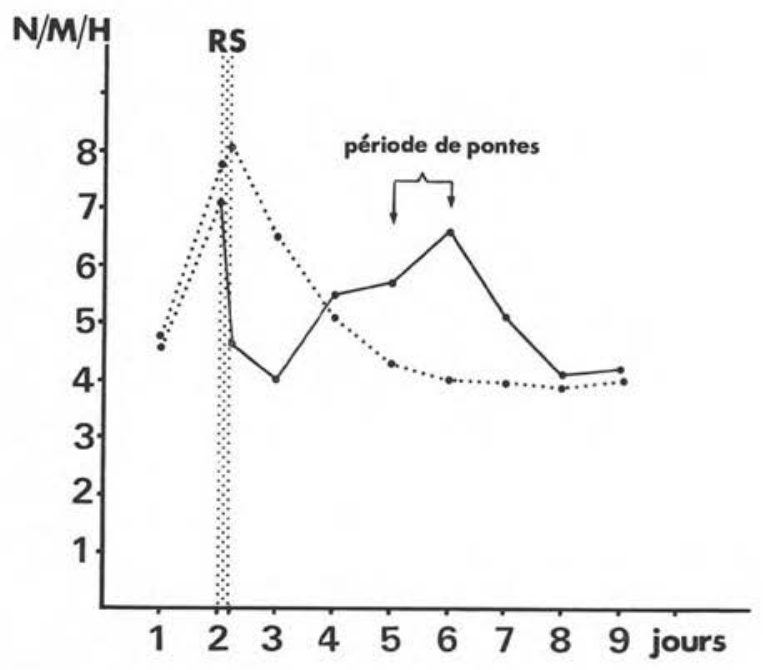

Fig. 3. Nombre d'accouplements chez les couples soumis à un flux d'air venant de 80 femelles vierges non nourries $(\ldots \ldots)$ et de 80 femelles vierges nourries (- - ). 


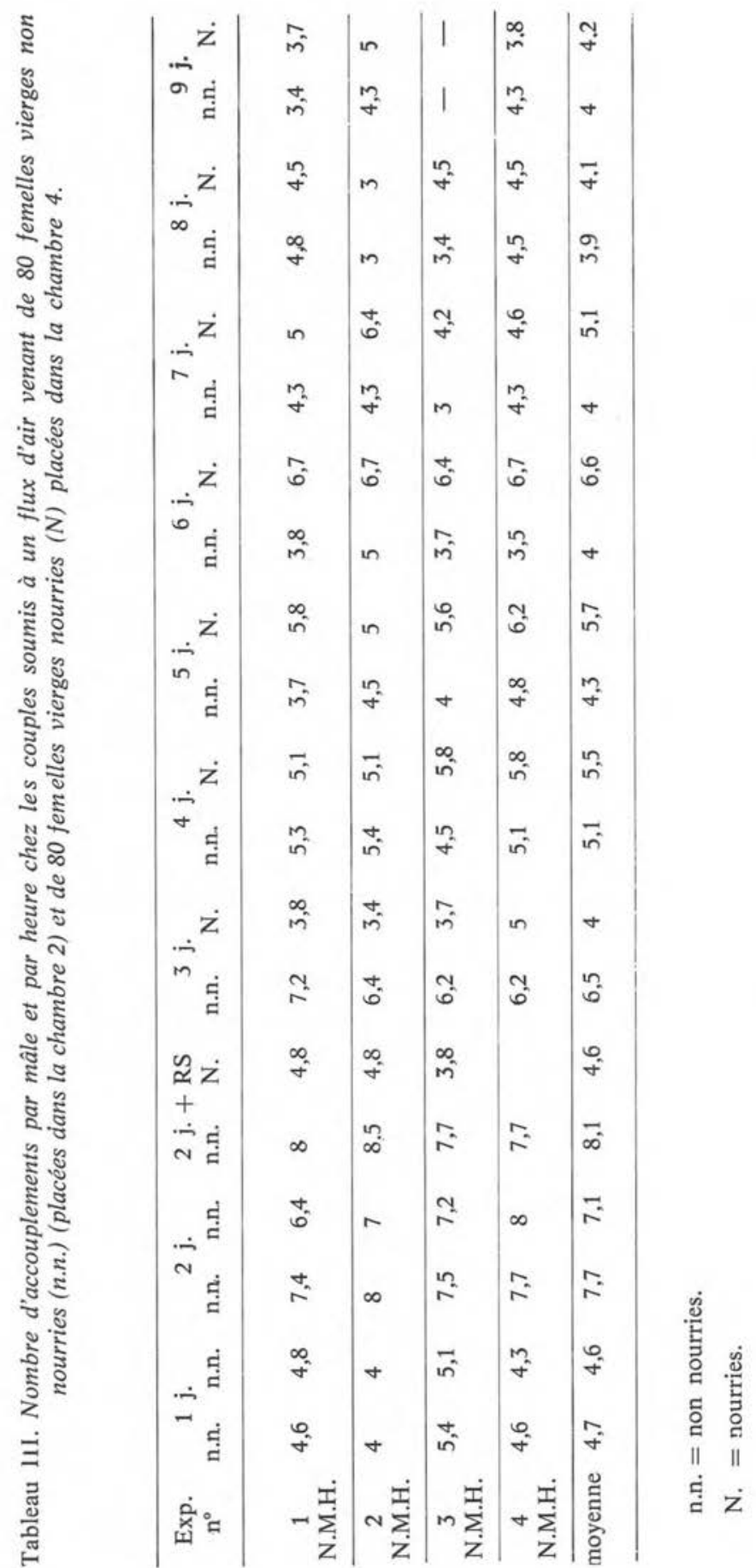




\section{Discussion et conclusion.}

L'action du repas sanguin ne semble pas modifier la sécrétion de phéromone puisque le nombre d'accouplements avant le repas et pendant le repas ne varie pas (tableau I).

Par ailleurs, nous avons constaté chez C. nubeculosus, en élevage au laboratoire, que la copulation pouvait se produire au moment où la femelle se gorgeait. Pomérantzev (1932), a observé le même phénomène. Par contre, après le repas sanguin, le $\mathrm{N} / \mathrm{M} / \mathrm{H}$ diminue sensiblement (tableau II).

Cette diminution reste évidente jusqu'à la $24^{e}$ heure après le repas, puis après 48 heures, nous constatons de nouveau une augmentation de la sécrétion de phéromone lorsque les œufs sont mûrs et, surtout, le pic devient plus évident pendant la période de ponte.

La sécrétion semble ensuite diminuer (tableau III).

Dans la série de femelles vierges, non nourries, le maximum de la sécrétion de phéromone est atteint au deuxième jour, puis on observe une diminution lente en fonction de l'âge des femelles. Ceci est à rapprocher des travaux de Roger et coll., (1969), qui ont observé également que la sécrétion de la phéromone chez les femelles vierges de Lucilia cuprina atteint le maximum au $4^{e}$ jour, puis diminue en fonction de l'âge des femelles.

\section{II. - Influence du repas sanguin sur l'effet attractif de la phéromone}

L'existence d'une phéromone sexuelle sécrétée par les femelles vierges de $C$. nubeculosus est démontrée par la série d'observations que nous avons rapportées dans une étude précédente. Nous avons constaté qu'elle augmente de façon très appréciable le nombre des copulations.

Nous avons précisé (Kremer et coll.), que cette substance est diffusible dans l'air selon un gradient et qu'elle a un rôle attractif. Nous étudions ici les variations de l'effet attractif de la phéromone sous l'influence du repas sanguin.

Nous avons utilisé le même dispositif décrit ailleurs (Kremer et coll.), pour mettre en évidence l'effet attractif de la phéromone : tube en verre en forme de $\mathrm{T}$, dont chacune des 3 branches mesure $20 \mathrm{~cm}$.

Les branches sont numérotées $\mathrm{B} 1, \mathrm{~B} 2, \mathrm{~B} 3$. Leur diamètre très régulier est de $1,5 \mathrm{~cm}$.

Aux deux extrémités des branches B1 et B2 nous disposons deux cylindres en verre d'une longueur de $2,5 \mathrm{~cm}$ recouverts des deux côtés de bas de nylon (cylindres $\mathrm{C} 1$ et $\mathrm{C} 2$ ) ; dans la branche 3, un cylindre (C3) ouvert d'un côté et dont l'autre extrémité est fixée sur une boucle avec une ficelle: montage réalisé selon la figure 4 . 


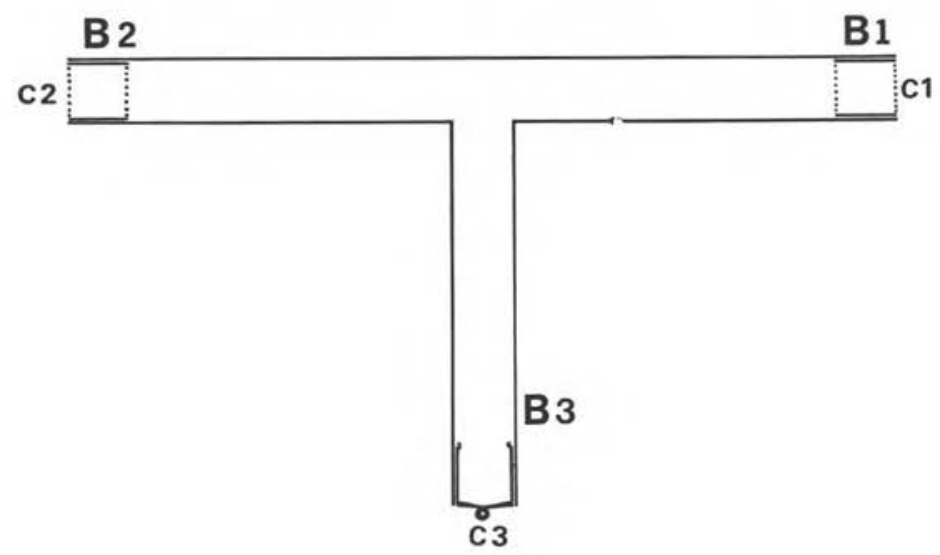

Fig. 4. Tube en $\mathrm{T}$ pour étudier l'effet attractif de la phéronome.

Nous pouvons également adapter aux extrémités des deux branches B1 et B2, des tubes reliés aux bocaux décrits dans l'étude précédente.

L'introduction des mâles par le cylindre 3 se fait après légère anesthésie au $\mathrm{CO}_{2}$.

Les expériences ont été faites à l'insectarium à $27^{\circ} \mathrm{C}$, avec 70 à $80 \%$ d'humidité relative et éclairage constant.

A. - L'effet attractif de la phéromone pendant le repas sanguin.

\section{Expérience 1:}

Nous avons mis dans les cylindres (C1 et C2), 50 femelles de Culicoides de 2 jours, vierges non nourries, puis nous avons lâché environ 70 mâles du même âge dans la branche 3 du tube en $\mathrm{T}$; nous avons ensuite compté le nombre de mâles se dirigeant vers les deux côtés en fonction du temps. Les résultats sont exprimés par la première partie du tableau $I V$ dans lequel le premier chiffre indique le nombre de mâles comptés dans $\mathrm{B} 1$ et le $2^{\mathrm{c}}$ ceux comptés en $\mathrm{B} 2 . \mathrm{C} 1 / \mathrm{C} 2$ indique que $\mathrm{C} 1$ est dans $\mathrm{B} 1$ et $\mathrm{C} 2$ dans $\mathrm{B} 2 . \mathrm{C} 2 / \mathrm{C} 1$ indique une disposition inverse.

\section{Expérience 2:}

Pour étudier l'attractivité des femelles pour les mâles pendant le repas sanguin, nous avons placé une jeune souris endormie par injection de Nembutal, contre le cylindre (C1) qui contient 50 femelles vierges. 
Dans le cylindre (C2), 50 femelles également vierges, mais sans souris, servent de témoin.

$\mathrm{Au}$ moment où nous étions certains que le repas sanguin des femelles avait commencé, nous avons lâché 70 mâles du même âge dans la branche 3 .

Le nombre de mâles se dirigeant vers les deux côtés a été noté comme précédemment et, en fonction du temps. Les résultats sont exprimés dans la deuxième partie du tableau 4. Les expériences 1 et 2 ont été faites 4 fois et chaque fois, nous avons changé la position des femelles $(\mathrm{C} 1 / \mathrm{C} 2$ ou $\mathrm{C} 2 / \mathrm{C} 1)$. Les mâles ont été endormis à chaque expérience au $\mathrm{CO}_{2}$

Tableau IV. "Nombre de mâles se dirigeant vers les côtés B1 et B2 du tube en $T$ en fonction $d u$ temps, avant et pendant le repas sanguin. Le cylindre C1 contient 50 femelles vierges. La deuxième partie du tableau donne les résultats après un repas sanguin. Le cylindre C2 contient 50 femelles également vierges qui servent de témoin et qui ne sont pas nourries. $C 1 / C 2$ indique que $C 1$ est dans $B 1$ et $C 2$ dans $B 2 ; C 2 / C 1$ indique une disposition inversée. »

\begin{tabular}{|c|c|c|c|c|c|c|c|c|}
\hline \multicolumn{2}{|c|}{$\begin{array}{l}\begin{array}{c}\text { Nombre } \\
\text { de }\end{array} \\
\text { mâles se } \\
\text { dirigeant } \\
\text { vers B1 et } \\
\text { B2 }\end{array}$} & $\begin{array}{c}\text { Temps en } \\
\text { minutes après } \\
\text { lachage } \\
\text { des } \\
\text { mâles }\end{array}$ & 1 & 2 & 3 & 5 & 10 & 15 \\
\hline avant & Expé & ence $1 \mathrm{C} 1 / \mathrm{C} 2$ & $1 / 1$ & $2 / 3$ & $4 / 5$ & $7 / 10$ & $13 / 13$ & $22 / 18$ \\
\hline le & Expé & ence $2 \mathrm{C} 1 / \mathrm{C} 2$ & $1 / 2$ & $2 / 2$ & $4 / 5$ & $7 / 11$ & $15 / 17$ & $16 / 19$ \\
\hline repas & Expé & ence $3 \mathrm{C} 2 / \mathrm{C} 1$ & $0 / 2$ & $3 / 2$ & $6 / 3$ & $8 / 7$ & $19 / 16$ & $22 / 18$ \\
\hline $\begin{array}{l}\text { gan- } \\
\text { guin }\end{array}$ & Expé & ence $4 \mathrm{C} 2 / \mathrm{C} 1$ & $1 / 1$ & $4 / 3$ & $4 / 6$ & $9 / 8$ & $22 / 20$ & $25 / 24$ \\
\hline pen- & Expé & ence $1 \mathrm{C} 1 / \mathrm{C} 2$ & $0 / 2$ & $2 / 4$ & $3 / 5$ & $7 / 10$ & $15 / 18$ & $23 / 26$ \\
\hline dant & Expé & ence $2 \mathrm{C} 1 / \mathrm{C} 2$ & $2 / 1$ & $6 / 5$ & $10 / 9$ & $15 / 17$ & $20 / 24$ & $23 / 28$ \\
\hline le & Expé & ence $3 \mathrm{C} 2 / \mathrm{C} 1$ & $2 / 2$ & $4 / 3$ & $5 / 8$ & $10 / 11$ & $19 / 17$ & $27 / 20$ \\
\hline $\begin{array}{l}\text { repas } \\
\text { san- } \\
\text { guin }\end{array}$ & Expé & ence $4 \mathrm{C} 2 / \mathrm{C} 1$ & $0 / 2$ & $5 / 3$ & $6 / 8$ & $10 / 8$ & $20 / 17$ & $22 / 20$ \\
\hline
\end{tabular}

Sur le tableau $I V$, nous constatons que la répartition des mâles dans les 2 branches du tube en $\mathrm{T}$ est sensiblement la même, avant le repas sanguin et pendant le le repas.

Celui-ci ne modifie donc pas l'attractivité des femelles pour les mâles.

Des expériences préliminaires avaient été faites pour étudier l'effet attractif que pourrait représenter l'odeur de la souris pour les mâles. Nous n'avons observé aucun effet. 


\section{B. - L'effet attractif de la phéromone trois heures après le repas sanguin.}

Nous prenons deux bocaux reliés au tube en T. Dans l'un (b1), nous plaçons 80 femelles vierges de deux jours, gorgées depuis 3 heures. Dans le $2^{\mathrm{e}}$ bocal (b2), nous plaçons 80 femelles vierges du même âge, non gorgées.

Le flux d'air passe pendant 3 minutes des bocaux vers les branches du $\mathrm{T}$, puis nous coupons le contact entre le tube en $\mathrm{T}$ et les bocaux.

Environ 90 mâles sont libérés dans la branche 3. Le nombre de mâles présents dans les 2 branches est ensuite compté.

L'expérience a été faite 5 fois en intervertissant la position des femelles. Le résultat est exprimé, comme précédemment, dans le tableau $I I$. b1/b2 signifie que le bocal b1 était relié à la branche $\mathrm{B} 1$ et le bocal b2 à la branche B2 du T. b2/b1 indique un branchement inversé.

Nous constatons que le nombre de mâles qui se trouve dans les deux branches 1 et 2 n'est pas identique, il est environ le double du côté où le flux d'air provient des femelles vierges non gorgées (b2). Ceci tend à montrer que les femelles vierges non nourries secrètent beaucoup plus de phéromone que les femelles du même âge gorgées depuis 3 heures (b1).

\section{C. - L'effet attractif de la phéromone après la première ponte.}

Nous prenons les mêmes bocaux que dans l'expérience précédente. Dans l'un, nous plaçons 80 femelles vierges de 6 jours qui ont pris un repas sanguin au $2^{\mathrm{e}}$ jour de leur vie imaginale et dont la plupart ont pondu au $5^{\mathrm{e}}$ jour (b1).

Dans le $2^{\mathrm{e}}$ bocal, nous plaçons 80 femelles vierges du même âge, à jeun (b2).

Nous établissons le flux d'air pendant 3 minutes, ensuite nous coupons le contact entre le tube en $\mathrm{T}$ et les bocaux. Une centaine de mâles est libérée dans la branche 3 . Nous comptons le nombre de mâles présents dans les deux branches après des temps croissants. L'expérience a été faite 4 fois en changeant à chaque fois la position des femelles.

Le résultat est donné par le tableau $V$.

Nous observons que le nombre de mâles qui se sont dirigés vers la branche où le flux d'air provient des femelles ayant pondu (b1), est plus élevé que du côté où le flux d'air provient des femelles du même âge, mais non gorgées (b2).

Nous pouvons constater que la maturation des œufs diminue la production de phéromone puisque la sécrétion reprend après la maturation.

\section{Discussion et conclusion.}

Pendant le repas sanguin, l'attractivité des femelles pour les mâles ne diminue pas immédiatement, les femelles continuent à produire de la phéromone comme l'indique le résultat du tableau I. Par contre, 3 heures après le repas, l'attractivité des femelles pour les mâles diminue de façon sensible $\left(1^{\text {re }}\right.$ partie du tableau $V$ ), et augmente de nouveau après la maturation de la ponte ( $2^{e}$ partie du tableau $\left.V\right)$. 
Tableau V. "Nombre de mâles se dirigeant vers les deux côtés du tube en $T$, (B1 et B2) sous l'influence du flux d'air provenant des bocaux (b1 et b2), en fonction du temps.

Pour la $1^{\text {re }}$ partie du tableau, le bocal b1 contient 80 femelles vierges de 2 jours gorgées depuis 3 heures, le bocal b2 contient 80 femelles vierges du même âge, non gorgées.

Pour la $2^{\star}$ partie du tableau, le bocal 1 contient 80 femelles vierges de 6 jours qui ont pondu et le bocal 2 contient des femelles de 6 jours, à jeun.

$b 1 / b 2$ indique que b1 est relié à la branche $B 1$ et b2 à $B 2 ; b 2 / b 1$ indique un branchement inverse. »

\begin{tabular}{|c|c|c|c|c|c|c|c|}
\hline $\begin{array}{c}\text { Nombre } \\
\text { de } \\
\text { mâles se } \\
\text { dirigeant } \\
\text { vers B1 et } \\
\text { B2 }\end{array}$ & $\begin{array}{c}\text { Temps en } \\
\text { minutes après } \\
\text { lâchage } \\
\text { des } \\
\text { mâles }\end{array}$ & 1 & 2 & 3 & 5 & 10 & 15 \\
\hline $\begin{array}{cc}3 \text { heures } & \mathrm{E} \\
\text { après le } & \mathrm{E} \\
\text { repas } & \mathrm{E} \\
\text { sanguin } & \mathrm{E} \\
& \mathrm{E}\end{array}$ & $\begin{array}{lll}\text { Expérience } & 1 & \mathrm{~b} 1 / \mathrm{b} 2 \\
\text { Expérience } & 2 & \mathrm{~b} 2 / \mathrm{b} 1 \\
\text { Expérience } & 3 & \mathrm{~b} 2 / \mathrm{b} 1 \\
\text { Expérience } & 4 & \mathrm{~b} 1 / \mathrm{b} 2 \\
\text { Expérience } & 5 & \mathrm{~b} 1 / \mathrm{b} 2\end{array}$ & $\begin{array}{l}2 / 0 \\
1 / 0 \\
0 / 1 \\
0 / 2 \\
2 / 1\end{array}$ & $\begin{array}{l}3 / 2 \\
5 / 2 \\
3 / 7 \\
1 / 4 \\
2 / 4\end{array}$ & $\begin{array}{c}4 / 8 \\
16 / 20 \\
- \\
13 / 10 \\
4 / 7\end{array}$ & $\begin{array}{r}6 / 25 \\
28 / 14 \\
17 / 17 \\
6 / 14\end{array}$ & $\begin{array}{l}9 / 41 \\
-70 / 20 \\
19 / 28 \\
11 / 23\end{array}$ & $\begin{array}{l}12 / 25 \\
48 / 27 \\
43 / 21 \\
20 / 28 \\
17 / 39\end{array}$ \\
\hline $\begin{array}{cc}48 \text { heures } & \mathrm{E} \\
\text { après la } & \mathrm{E} \\
\text { ponte } & \mathrm{E} \\
& \mathrm{E}\end{array}$ & $\begin{array}{lll}\text { Expérience } & 1 & \mathrm{~b} 1 / \mathrm{b} 2 \\
\text { Expérience } & 2 & \mathrm{~b} 1 / \mathrm{b} 2 \\
\text { Expérience } & 3 & \mathrm{~b} 2 / \mathrm{b} 1 \\
\text { Expérience } & 4 & \mathrm{~b} 2 / \mathrm{b} 1\end{array}$ & $\begin{array}{l}1 / 0 \\
2 / 1 \\
1 / 1 \\
0 / 1\end{array}$ & $\begin{array}{l}4 / 1 \\
5 / 3 \\
2 / 4 \\
2 / 4\end{array}$ & $\begin{array}{r}7 / 3 \\
13 / 5 \\
6 / 9 \\
5 / 2\end{array}$ & $\begin{array}{l}9 / 3 \\
30 / 11 \\
15 / 20 \\
8 / 16\end{array}$ & $\begin{array}{l}23 / 15 \\
49 / 28 \\
24 / 28 \\
22 / 32\end{array}$ & $\begin{array}{l}51 / 13 \\
50 / 26 \\
45 / 30 \\
24 / 33\end{array}$ \\
\hline
\end{tabular}

Ce résultat confirme ce que nous avons déjà observé lors de la première partie de ce travail sur l'effet stimulant sur l'accouplement de la phéromone. Des expériences, actuellement en cours, montrent qu'après cette augmentation, la phéromone est à nouveau de moins en moins produite.

\section{Bibliographie}

Bartell R. J., Shorey H.H., Barton Browne J.: Pheromonal stimulation of the sexual activity of males of the sheel blowfly Lucilia cuprina by the female. Anim. Behav., 1969, 17, 576-585.

Boorman J.: The maintenance of laboratory colonies of Culicoides variipennis (Coq.), C. nubebulosus (mg) and C. riethi (Kieff.) (Diptera, Ceratopogonidae). Bull. Ent. Res., 1974, 64, 371-377.

Downes J. A.: Observation on the swarming flight and mating of Culicoides (Diptera, Ceratopogonidae). Trans. R. Ent. Soc. Lond., 1955, 106, 213-236.

Kremer M., Ismail M.T., Rebholtz C.: Detection of a pheromone released by the females of Culicoides nubeculosus (Diptera, Ceratopogonidae) attracting the males and stimulating copulation. Mosquito News, 1979, 39, 627-631.

Pomerantzev B. I.: Beitrage zur Morphologie und Anatomie der genitalen von Culicoides (Diptera, Nematocera). Mag. Paras. Inst. Zool. Acad. Sci. URSS, 1932, 3, 183-214. 